\title{
Quality Control at the U.S. Geological Survey National Water Quality Laboratory
}

\section{INTRODUCTION}

The National Water Quality Laboratory (NWQL)-the primary analytical facility of the U.S. Geological Survey (USGS)-analyzes environmental samples from across the United States and around the world. Samples of water, sediment, aquatic biological materials, and air are analyzed at the laboratory using state-of-the-art instruments and methods. These analyses require a comprehensive qualityassessment process.

Quality assessment consists of two separate but related components: quality-control data and quality-assurance elements. Quality-control (QC) data are produced to measure bias and variability of the analytical method. Without QC data, the magnitude of errors, and thus the quality of the environmental data, is not known. Some examples of QC data sample types include standard reference materials, laboratory replicates, laboratory reagent blanks, spikes, and surrogates. Assessment of QC data is essential to evaluating environmental analytical data produced by a laboratory. Definitions of QC data sample types used by the NWQL are emphasized in this Fact Sheet.

Quality-assurance (QA) elements are also essential to producing high-quality analytical results. QA elements are defined as the procedures used to control the unmeasurable components of the analytical method. QA elements include standard operating procedures, analysis-sequence protocols, instrument-maintenance logs, and personnel training records. Clearly, all of these elements could affect laboratory data quality. However, it is often difficult to ascertain the impact of QA elements on environmental analytical results. Therefore, the assessment of QA elements will not be discussed in this Fact Sheet.

Quality assessment is the process of reviewing the application of QA elements and the analysis of QC data. The objective of quality assessment is to provide evidence that the measurement processes are under control.

\section{QUALITY-CONTROL PROCESS}

The NWQL uses a three-tiered approach to QC divided into inorganic and organic analyses. For the first tier, "Method Performance," QC samples are analyzed by a chemist. At this level, chemists use the QC sample data to control the analytical process for each batch of environmental samples. If prescribed acceptance criteria are not met, the analysis is discontinued until corrected.

The second tier contains the "Data Review and Blind Sample Programs." Chemists use data from these programs to monitor method performance throughout the laboratory and over long periods.

The third and final tier consists of NWQL's participation in "Performance-Evaluation Studies." These studies are managed by local, State, or Federal agencies external to the NWQL. Data from these studies can be used to compare laboratories or to select a laboratory for analytical work. Summary reports from many of these studies are available on the NWQL Home Page Website at wwwnwql.cr.usgs.gov/USGS.

\section{INORGANIC ANALYSIS}

\section{First Tier: Method Performance}

Analysts trying to determine inorganic constituents in any environmental sample evaluate two areas of performance: instrument and method. Accurate instrument operation can be verified by analyzing known standards at different concentrations. Inorganic blank water, which has negligible concentrations of the constituents of interest, is also analyzed to evaluate and eliminate potential sources of bias.

The analyst uses QC samples to assess whether the method is working properly. Generally, data produced from these samples are the most useful for customers to evaluate the entire analytical process. In addition to inorganic blank water samples, standard reference materials are the primary tools for evaluating method bias and variability. Laboratory replicate data also might be evaluated to obtain method variability information. Definitions of these sample types follow.

Standard Reference Materials (SRMs). Generally, SRMs are supplied in one of two ways: as an ampoule in concentrated form that is added to the appropriate matrix, or as a prepared reference ready for analysis. The prepared reference is the predominant source when commercial materials are 
available. In either form, the SRM is thought to be sufficiently tested to be used to assess method performance. Several SRMs of differing concentrations and constituents are analyzed alongside environmental samples at the NWQL. The SRM data are evaluated to assess bias for each analysis. Additionally, as the data are compiled, long-term estimates of method variability can be obtained.

Laboratory Replicate. A laboratory replicate is one type of environmental sample replicate. The environmental sample is split at the laboratory to provide information about variability. If the sample is split into identical subsamples, then the replicate will provide information about variability inherent in the analytical process. However, true sample splits are often difficult to obtain. More frequently, the split samples will exhibit some differences because of sample inhomogeneity. The sample variability inherent in more challenging matrices, such as sediment, will often mask the sample processing and analytical variability.

\section{Second Tier: Data Review and Blind Sample Programs}

Data Review Program. All inorganic analytical results initially are stored in the Laboratory Information Management System data base. A QC check program uses chemical logic checks to review all analytical results in the data base. All analytical results that fall outside the acceptance limits are sent to chemists in the Quality Assurance Unit for review. Examples of these logic checks include the following:

- Cation/Anion Balance

- Specific Conductance/Anion Ratio

- Specific Conductance/Cation Ratio

- Dissolved Solids/Specific Conductance Ratio

- Field Value/Laboratory Value Agreement (pH, alkalinity, specific conductance)

- Filtered Component Compared with Unfiltered Component

Blind Sample Programs. The inorganic blind sample programs are administered by two different organizations, one internal and the other external to the NWQL. The internal program quantifies bias caused by random laboratory contamination. It also supplements the external blind sample program by submitting double-blind reference samples for those analyses not evaluated externally (cyanide, whole water recoverable constituents, sediment, tissue). These reference samples are called "double blind" because both the sample origin and constituent concentrations are unknown to the analyst.

The external blind sample program is used to evaluate both the NWQL and the USGS laboratory in Ocala, Fla. Bias and variability information obtained from this program is made available to laboratory customers on the Web at

$$
\text { wwwbtdqs.cr.usgs.gov. }
$$

Data analysis performed by either program typically involves preparing control charts to monitor long-term method performance. The number of standard deviations from the most probable value (otherwise known as the $\mathrm{z}$-value) is calculated. Using this calculation, all data can be easily displayed in a single chart. The $\mathrm{z}$-value is calculated as follows:

laboratory measured concentration - most probable value standard deviation of most probable value

In figure 1 , the $\mathrm{z}$-values calculated for cadmium represent concentrations ranging from 0 to 50 micrograms per liter.

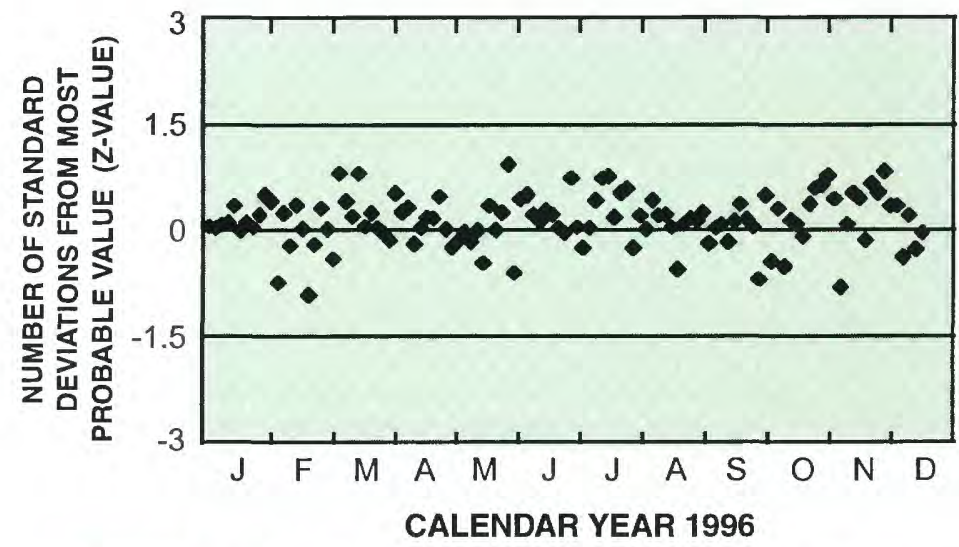

Figure 1. Control chart of cadmium determinations in standard reference material samples by graphite furnace atomic absorption spectrophotometry.

\section{Third Tier: Performance-Evaluation Studies}

The NWQL participates in the following national and international interlaboratory performance-evaluation studies:

- U.S. Environmental Protection Agency (USEPA) WaterPollution (WP), Water-Supply (WS), and Safe Drinking Water Act (radiochemistry) studies;

- USGS Branch of Quality Systems Evaluation Program for Standard Reference Samples; 
- National Water Research Institute of Canada; and

- National Oceanic and Atmospheric Administration (NOAA) Intercomparison Program for Marine Sediment and Tissue.

\section{Field Supplies}

The USGS warehouses stock sample containers, filters, and preservatives for collecting environmental samples. Inorganic blank water is also available for customer use. All supplies are rigorously tested to ensure compliance with quality standards. Contamination is tested using the lowest detection methodology for the constituents of interest. Certificates of analysis are provided with each manufacturer's lot. Other field QC supplies, such as inorganic spike materials, are available on request.

\section{ORGANIC ANALYSIS}

\section{First Tier: Method Performance}

Organic compounds may be degraded by heat, light, temperature, microorganisms, and other factors. Because the NWQL analyzes a wide range of samples and specializes in trace and ultratrace compound concentrations, there is an increased need for QC information compared to inorganic analyses.

Samples received for organic determinations are grouped together for similar analyses. Laboratory reagent blanks and laboratory reagent spike samples are prepared and analyzed with each group of environmental samples to monitor method performance. Surrogate compounds are also added to all environmental and QC samples. These QC sample types are defined as follows.

Laboratory Reagent Blank. The laboratory reagent blank (LRB) is prepared using an appropriate matrix. The selected matrix should have negligible concentrations of compounds for that particular analysis. The LRB is processed in the same way as the accompanying environmental sample and so is used to monitor for random contamination introduced from laboratory processing. If a compound is detected and identified in the LRB, quantitation of that same compound in the environmental sample would be qualified. Not only does the analyst track LRB results with each sample set, but the laboratory can track a history of LRB results to determine possible trends.

Laboratory Reagent Spike. A laboratory reagent spike (LRS) is prepared and analyzed with each group of environmental samples. The LRS is a sample where known concentrations of the compounds of interest are added, usually into a synthetic (reagent) matrix. An evaluation of reagent spike data yields information about method performance. The LRS analytical results are compared to acceptance criteria for each method to assess potential bias of environmental sample results. In addition, LRS data are charted periodically to observe long-term method bias and variability. A graph of the percent recovery of the herbicide atrazine, an organic compound in the LRS, is shown in figure 2 .

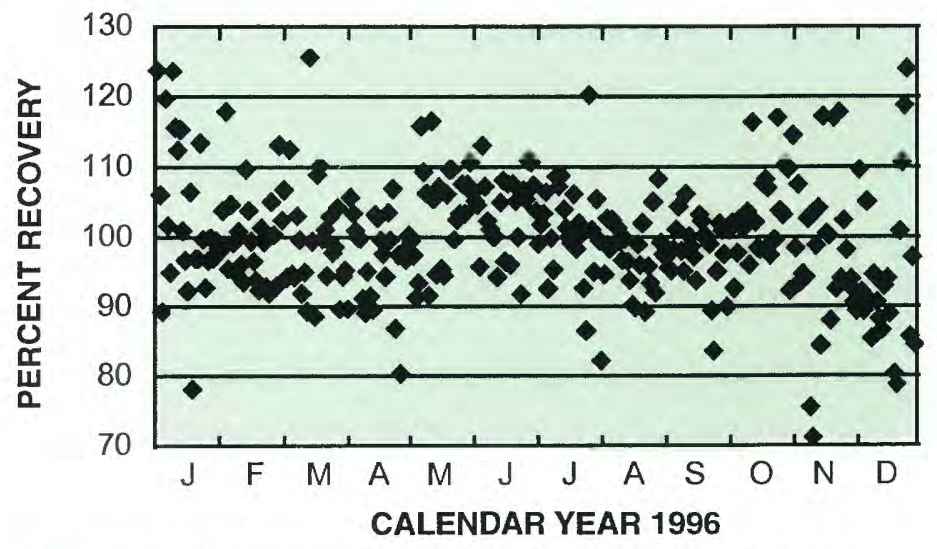

Figure 2. Percent recovery of the herbicide atrazine analyzed by solid-phase extraction, gas chromatography/ mass spectrometry.

Surrogate. A surrogate is a compound that is expected to perform similarly to the compounds being analyzed in a laboratory method. The surrogate is not normally found in the environment and therefore can be used to monitor the recovery efficiency of analytical processes. The NWQL adds surrogate compounds to all environmental and QC samples to monitor compound recovery and potential environmental effects. For example, high dissolved organic carbon concentrations might interfere with analytical techniques. Surrogate analytical results are released electronically with environmental data for all organic analyses.

\section{Second Tier: Blind Sample Program and Data Review}

Blind Sample Program. The first major component within the second tier of quality control is the blind sample program where double-blind samples are submitted for analysis. Results of double-blind samples should accurately reflect the state of laboratory processes since the samples do not receive special treatment.

Data Review. Another major QC activity is long-term dataquality assessment. This type of data review involves evaluating control charts and analyzing statistics from the LRBs, LRSs, and surrogates. 
Several statistical techniques are used for data analysis. Results are used to compute long-term control limits, which, in turn, are used to produce new acceptance criteria. Frequently, a control limit is set at three times the standard deviation of the expected value. If only normal random errors are present, then 99.7 percent of the values will fall within this limit (Friedman and Erdmann, 1982, p. 91).

\section{Third Tier: Performance-Evaluation Studies}

Performance-evaluation studies for organic compounds are more difficult to administer than for inorganic constituents because some of the compounds are unstable. Special care is required to ensure that performance-evaluation studies evaluate method performance and are not biased by natural compound degradation. The special measures that must be taken (for example, chilling samples and protecting them from light), along with the high cost of organic sample analysis, can make the studies expensive. Nevertheless, the NWQL routinely participates in the following national performance-evaluation studies:

- USEPA Water-Pollution (WP) and Water-Supply (WS) studies,

- NOAA Intercomparison Program for Marine Sediment and Tissue, and

- National Water Research Institute of Canada (organic carbon).

\section{Field Supplies}

The USGS warehouses stock the necessary supplies for collecting environmental samples for organic analyses, including sample containers, preservatives, filters, and solid-phase extraction cartridges. In addition, organic blank water suitable for pesticide and volatile analyses is available. Blank water is rigorously tested and quality assured at the laboratory and is distributed with a certificate of analysis. Complete field spike kits, consisting of a volumetric measuring device, necessary solvents, gloves, and a waste storage container, can be purchased from the NWQL. Verified spike solutions for several compound classes are prepackaged and ready for use. Custom spike solutions tailored to meet field QC activities also can be prepared.

\section{SELECTED REFERENCES}

Fishman, M.J., ed., 1993, Methods of analysis by the U.S. Geological Survey National Water Quality LaboratoryDetermination of inorganic and organic constituents in water and fluvial sediments: U.S. Geological Survey Open-File Report 93-125, 217 p.

Fishman, M.J., and Friedman, L.C., 1989, Methods for determination of inorganic substances in water and fluvial sediments: Techniques of Water-Resources Investigations of the U.S. Geological Survey, book 5, chap. A1, 545 p.

Friedman, L.C., and Erdmann, D.E., 1982, Quality assurance practices for the chemical and biological analyses of water and fluvial sediments: U.S. Geological Survey Techniques of Water-Resources Investigations, book 5, chap. A6, $181 \mathrm{p}$.

Pritt, J.W., and Raese, J.W., 1995, Quality assurance/quality control manual-National Water Quality Laboratory: U.S. Geological Survey Open-File Report 95-443, 35 p.

Wershaw, R.L., Fishman, M.J., Grabbe, R.R., and Lowe, L.E., 1987, Methods for the determination of organic substances in water and fluvial sediments: Techniques of Water-Resources Investigations of the U.S. Geological Survey, book 5, chap. A3, 80 p.

- Kimberly D. Pirkey and Stephen R. Glodt

For more information, contact:

Chief, Branch of Analytical Services

USGS, National Water Quality Laboratory

Box 25046, Mail Stop 407

Denver, Colorado 80225 